\title{
Deutsch für Fortgeschrittene
}

\section{Erhard Taverna}

Dr. med., Mitglied der Redaktion

Mark Twain tat sich schwer mit dem Erlernen der deutschen Sprache. In seinem satirischen Essay von 1880 «The Awful German Language» machte er zahlreiche Verbesserungsvorschläge. Dazu gibt es eine schöne Anekdote. Twain hörte sich im Reichstag in Berlin eine Rede Bismarcks an. "Was sagt er denn?», fragte er seinen Dolmetscher. «Moment.» flüsterte dieser. Und als Twain noch einmal nachfragte, sagte sein Dolmetscher: «Ich warte immer noch auf das Verb.» Die Syntax ist der Schrecken der Deutschlernenden. Twain behauptete, man lerne Französisch oder Englisch in dreissig Tagen, Deutsch aber in dreissig Jahren. In Wahrheit kannte er sich in dieser Sprache recht gut aus. Dabei ist die perfekte Beherrschung des Englischen äusserst schwierig, was mit dem grossen Anteil an Redewendungen zu tun hat. English is the easiest language to speak badly, besagt eine alte Redensart. Genau genommen sind das Hochenglische und die in-

Twain behauptete, man lerne Französisch oder Englisch in dreissig Tagen, Deutsch aber in dreissig Jahren.

ternationale Verkehrssprache, vor allem der Wissenschaften, des Internets und der Pop-Kultur zwei verschiedene Hüte. Die Qualität eines native speakers wird von den Wenigsten erreicht. Das Englische wird durch den inkompetenten globalen Gebrauch umgeformt und das wahrscheinlich stärker als die deutsche Sprache. Solange Amerika der Innovationsmotor der Welt bleibt, wird die simplifizierte Variante des Englischen, auch Globish genannt, die unverzichtbare Kommunikationssprache aller Nationen bleiben. Wir können sagen, dass wir einfach verschiedene Sprachen für verschiedene Ziele einsetzten.

Aus deutschsprachiger Sicht werden oft der Sprachverfall und Niedergang des Deutschen beklagt. Dafür gibt es zahlreiche Beispiele, aber ebenso viele, die das Gegenteil belegen. Mokieren kann man sich über das Imponiergehabe unnötiger Anglizismen in der Werbung und Wirtschaft, die unsere Alltagssprache durchsetzen. Jede Sprache wandelt sich. Auch der englische Wortschatz hat sich im Laufe der Geschichte sehr viele französische und deutsche Begriffe einverleibt. Eingewanderte beeinflussen unser Deutsch mit neuen
Sprachbildern und Wortkombinationen. Andreas Hock, der deutsche Journalist und Bestsellerautor, hat das in seinem Buch «Bin ich denn der Einzigste hier, wo Deutsch kann?» vielseitig und humorvoll beschrieben. Der Untertitel «Über den Niedergang unserer Sprache» ist mit einem Augenzwinkern zu verstehen. Ein unterhaltendes Buch, in dem der Autor häufig ausgestorbene, alte Begriffe im modernen Jugendslang entdeckt. Zum Beispiel das Wort lustwandeln, das noch in die Goethezeit verweist. Plötzlich taucht es wieder auf: «Bin total blass, und geh mal lustwandeln, um ein paar Pigmente zu haschen.» Ein ausführliches Glossar rundet diese Fundgrube an neuen Verwendungen ab. Am 21. Februar wird alljährlich der von der UNESCO aufgerufene «Tag der Muttersprache» begangen. Der Tag soll daran erinnern, dass Sprache kulturelle Identität und Denkheimat bedeutet. Wo Sprache ausserhalb des Alltags eine erkenntnisleitende Funktion hat, also vor allem in der Literatur und in den Geisteswissenschaften, sollte das einleuchten. Ohne solide Kenntnis der Muttersprache ist ein klares Denken nicht möglich. Wenn Anträge auf Fördermittel innerhalb einer deutschsprachigen Universität Englisch verfasst werden müssen, ist das ein Armutszeichen. Einerseits gibt es gewaltige Anstrengungen Zuwanderer vermittels Deutschkursen zu integrieren, andererseits schickt eine ambitionierte Elite ihre Kinder auf englischsprachige Gymnasien und Hochschulen. Wozu sollen Immigrantenkinder Deutsch lernen, wenn die Arbeitssprache Englisch ist? Und könnte es sein, dass auch ein linguistischer Graben die Kluft zwischen Normalos und denen da oben vertieft?

Zum Glück haben wir immer noch den Reichtum der Dialekte. Sie sind der Humus einer wunderbaren Hochsprache. Rap und Slam Poetry sind die neuen Triebe am alten Stamm. Goethe hat das so formuliert: «Die Gewalt einer Sprache ist nicht, dass sie das Fremde abweist, sondern dass sie es verschlingt.» Dem ist auch 200 Jahre später nichts beizufügen.

\section{Literatur}

Bin ich denn der Einzigste hier, wo Deutsch kann?

Andreas Hock, riva Verlag, 6. Auflage 2016. 